\title{
TRATAMIENTO CON HORMONA DE CRECIMIENTO Y CRANEOFARINGIOMA: PRESENTACIÓN DE CASO
}

\section{Treatment with growth hormone and craniopharyngioma: case presentation}

\author{
Dr. Francisco Carvajal Martínez, PhD*y Dr. Jorge Luis Bartolomé Copa**
}

Recibido: 2/8/2017 Aprobado: 2/9/2017

DOI: http://dx.doi.org/10.22206/cysa.2018.v2i1.pp15-20

\section{Resumen}

El craneofaringioma es el tumor más frecuente de la edad pediátrica. Se localiza frecuentemente en el área supraselar; histológicamente benigno, pero con carácter expansivo que provoca manifestaciones clínicas secundarias a la afectación de estructuras vecinas. El tratamiento es quirúrgico y, en la mayoría de los casos, trae como consecuencia un panhipopituitarismo. El tratamiento con hormona de crecimiento $(\mathrm{GH})$ en estos casos es un tema de debate. A nivel internacional se ha realizado en situaciones excepcionales, obteniendo resultados favorables. En Cuba, este es el primer caso dentro de una casuística de 813 pacientes tratados con $\mathrm{GH}$, en 12 años de experiencia.

Describimos el caso clínico de una paciente con diagnóstico de craneofaringioma, operada, que recibió tratamiento con hormona de crecimiento. Se trata de una fémina que a los 9 años de edad inicia sus estudios por presentar talla baja. Se le realizó test de clonidina que reveló déficit de GH. En la tomografía computarizada de cráneo se observó lesión hipofisaria de $2 \mathrm{~cm}$ de diámetro que ocupa cisterna supraselar, con lo que se llega al diagnóstico de un craneofaringioma. Se lleva a cabo la intervención quirúrgica con resección total macroscópica del tumor. Posteriormente, se instaura un panhipopituitarismo, por lo que se realiza sustitución del eje tiroideo, adrenal, gonadal y hormona antidiurética. Se evalúa alteración del crecimiento, ausencia de recidiva tumoral y, con el consentimiento de

\footnotetext{
* Departamento de Endocrinología Pediátrica, Instituto Nacional de Endocrinología (INEN), La Habana, Cuba. Endoped@ infomed.sld.cu

** Médico residente (segundo año), Instituto Nacional de Endocrinología (INEN), La Habana, Cuba.
}

\section{Summary}

Craniopharyngioma is the most frequent tumor of the pediatric age. It is frequently located in the suprasellar area; histologically benign, but with expansive character that causes clinical manifestations secondary to the involvement of neighboring structures. The treatment is surgical and, in most cases, results in panhypopituitarism. The treatment with growth hormone $(\mathrm{GH})$ in these cases is a subject of debate. At an international level, it has been carried out in exceptional situations, obtaining favorable results. In Cuba, this is the first case in a casuistry of 813 patients treated with GH, in 12 years of experience.

We describe the clinical case of a patient with a diagnosis of operated craniopharyngioma who received treatment with growth hormone. This is a female who, at 9 years of age, starts her studies because she has short stature. A clonidine test was performed, which revealed $\mathrm{GH}$ deficiency. In the cranial computed tomography, a pituitary lesion $2 \mathrm{~cm}$ in diameter was observed that occupies a suprasellar cistern, which leads to the diagnosis of a craniopharyngioma. Surgical intervention is carried out with macroscopic total resection of the tumor. Subsequently, a panhypopituitarism is established, which is why the thyroid, adrenal, gonadal and antidiuretic hormone are replaced. Growth alteration, absence of tumor recurrence is evaluated and, with the 
la familia, se inicia tratamiento con $\mathrm{GH}$, con lo que se obtiene ganancia de talla favorable de $39 \mathrm{~cm} / 5$ años, sin evidencia de recidiva tumoral.

Palabras clave: craneofaringioma; hormona de crecimiento; hipopituitarismo.

\section{Introducción}

El craneofaringioma es el tumor hipotalámico más frecuente de la infancia y constituye entre $80-90 \%$ de los tumores de esta región; se origina de restos de la bolsa de Rathke y se caracteriza por su componente quístico y por la presencia de calcificaciones $^{1,2}$.

Es un tumor histológicamente benigno, su localización habitual es en la región selar/ paraselar, es de crecimiento lento y tiene una fuerte tendencia a la expansión tumoral que afecta estructuras vecinas y lo convierten en un tumor con consecuencias clínicas graves ${ }^{1,3,4,5}$. El cuadro clínico es secundario a lesión hipotalámica, hipertensión endocraneana, lesión de nervio óptico e hipopituitarismo ${ }^{6}$.

Entre un $80-90 \%$ de los niños afectados presenta hipopituitarismo, siendo la deficiencia de hormona del crecimiento $(\mathrm{GH})$ y gonadotropinas las más frecuentes ${ }^{1}$. En niños, el déficit de GH ocasiona retraso del crecimiento, pero algunos conservan la velocidad de crecimiento normal, a pesar de un déficit completo de la hormona del crecimiento $^{6}$.

El tratamiento consiste en la extirpación quirúrgica del tumor $\mathrm{y}$, en algunos casos, la radioterapia. En la mayoría de los pacientes, tanto el efecto compresivo tumoral como el tratamiento quirúrgico traen como consecuencia un panhipopituitarismo ${ }^{7}$.

Con relación al déficit de $\mathrm{GH}$, el principal objetivo del tratamiento con hormona de crecimiento es la mejoría de la talla hasta alcanzar una talla adulta consent of the family, treatment with GH is initiated, obtaining a favorable height gain of $39 \mathrm{~cm} / 5$ years, without evidence of tumor recurrence.

Keywords: craniopharyngioma; growth hormone; hypopituitarism.

final normal, lo más aceptable dentro del potencial de crecimiento genético. La dosis y la posología han variado con el tiempo; actualmente la dosis para niños con déficit de $\mathrm{GH}$ presenta un rango entre $0.16-0.23 \mathrm{mg} / \mathrm{kg} / \mathrm{semana}^{8,9}$.

Eventos adversos a corto plazo han sido reportados en el tratamiento con hormona de crecimiento, tales como leucemia, pseudotumor cerebral, epifisiolisis de cadera, cefaleas, retención hídrica y Síndrome del túnel carpiano, entre otros ${ }^{10-12}$. Es muy difícil establecer la frecuencia de recidivas de tumores del sistema nervioso central; esta oscila entre $16,4 \%$ y $19,3 \%{ }^{1}$.

Se ha descrito que los pacientes operados de craneofaringioma tienen una elevada tasa de recidiva y progresión. En un estudio de 10 años de seguimiento a 126 pacientes con craneofaringioma operado, se observó una tasa de recidiva y progresión del $43 \%{ }^{5-7}$.

Por el interés clínico que representa la obtención de resultados favorables con relación al tratamiento con GH, en pacientes con déficit de GH secundario a craneofaringioma operado, es que se realiza la presentación del siguiente caso clínico.

\section{Caso clínico}

Paciente femenina producto de parto eutócico, a término, con buen peso y APGAR al nacer. A los 9 años de edad inició sus estudios por presentar talla baja, se determinó edad ósea de 6-7 años de edad, 
se realizó un test de clonidina que reveló valores muy bajos de $\mathrm{GH}$ :

$-30^{\prime}$ (minutos): $0.28 \mathrm{ng} / \mathrm{ml}$

$0^{\prime}: 0.30 \mathrm{ng} / \mathrm{ml}$

$60^{\prime}: 0,33 \mathrm{ng} / \mathrm{ml}$

$90^{\prime}: 0,29 \mathrm{ng} / \mathrm{ml}$

En la tomografía computarizada de cráneo (TAC) se observó lesión hipofisaria de $2 \mathrm{~cm}$ de diámetro que crece ocupando cisterna supraselar, por lo que se llegó al diagnóstico de craneofaringioma.

Se lleva a cabo la intervención quirúrgica donde se verificó la presencia de craneofaringioma y se le realizó resección total macroscópica del tumor. Posterior a la intervención quirúrgica, se instauró un panhipopituitarismo con afectación de los ejes; somatotropo, gonadotropo, tirotropo, corticotropo (Cortisol $11.9 \mathrm{nmol} / \mathrm{L}, \mathrm{FSH} 0.76 \mathrm{UI} / \mathrm{L}, \mathrm{LH}$ indetectable, TSH $1.7 \mathrm{mUI} / \mathrm{L}$, T4 $32,5 \mathrm{nmol} / \mathrm{L}$ ) y desarrollo de una diabetes insípida central vasopresina sensible. Por tal razón, se inició tratamiento sustitutivo con:

Acetato de cortisona $25 \mathrm{mg} /$ día, vía oral. Levotiroxina sódica $50 \mathrm{mg} / \mathrm{d}$ áa, vía oral.

DDAVP (1-desamino-8-D-arginidavasopresina): 10 ug cada 12 horas, vía nasal.

Se realizó TAC de control a los 6 meses y a los 4 años del tratamiento quirúrgico, que evidenció silla turca y sistema ventricular de dimensiones normales, sin constatar recidiva tumoral; también se realizó examen oftalmológico que resultó normal. En las consultas de seguimiento se observó que durante 4 ańos solo aumentó $2 \mathrm{~cm}$ de talla.

La ausencia de recidiva tumoral, la confirmación del déficit de $\mathrm{GH}$ con el test de clonidina, la deficiente ganancia de talla, la valoración por neurocirugía y el consentimiento de la familia, motivaron a iniciar tratamiento con GH a la edad de 13 7/12 años a dosis de $1 \mathrm{mg} / \mathrm{m}^{2} \mathrm{sc} / \mathrm{d}$ ía, con una talla inicial de $114 \mathrm{~cm}$ inferior al tercer percentil de la talla para la edad.
En las consultas de seguimiento se evaluó la curva de crecimiento, observándose buena respuesta al tratamiento con $\mathrm{GH}$, presentando una velocidad de crecimiento en los dos primeros ańos de 11-12 cm/año; en el tercer año de tratamiento la velocidad bajó a 7-8 cm/año y en los años posteriores a $2-3 \mathrm{~cm}$ por ańo, ganando en total $39 \mathrm{~cm}$ de talla desde los 13 años hasta los 18 años de edad, con lo que llega a situarse entre un 10-25 percentil para su edad. Además, se incorporó al tratamiento integral hormonas sexuales femeninas; en la actualidad, tiene un desarrollo sexual estadio $\mathrm{V}$ de TANNER.

El seguimiento fue realizado por un equipo multidisciplinario, constituido por un endocrinólogo pediatra, un neurocirujano y un oftalmólogo. Durante este seguimiento se observó buena tolerancia al medicamento, no se evidenció reacciones adversas ni activación tumoral, hubo cumplimiento del tratamiento por parte de la paciente y de los familiares, lo que contribuyó con la respuesta obtenida. Posteriormente, la paciente logró incorporarse a su vida social normal, tiene pareja, con relaciones sexuales normales, y estudió periodismo en la universidad.

\section{Discusión}

Existen pocos estudios que comparan la tasa de progresión tumoral en pacientes operados de craneofaringioma, con y sin tratamiento con $\mathrm{GH}$, entre los que se encuentran los reportados en las series de la National Cooperative Growth Study (NCGS) y la Pfizer International Growth Database (KIGS).

En la serie $\mathrm{NCGS}^{22}$ se publicó un análisis de reacciones adversas al tratamiento con $\mathrm{GH}$, donde se observó un $6,4 \%$ de recidiva tumoral en pacientes operados de craneofaringioma.

En el estudio retrospectivo realizado por el $\mathrm{KIGS}^{10,23,24}$, en 2,503 pacientes supervivientes de tumores cerebrales, entre ellos 1,038 con 
craneofaringioma, no encontraron un incremento en la tasa de recidivade estostumores, comparada con la obtenida de estudios de pacientes no tratados con GH $(11,4 \%$ en craneofaringiomas y $<10 \%$ para el resto). Tampoco encontraron que las recidivas tuvieran relación con las dosis de GH.

Karavitaki et al. ${ }^{25,26}$ realizaron un análisis retrospectivo de 32 pacientes con craneofaringioma que recibieron tratamiento con $\mathrm{GH}$, captados en un período de 21 ańos, y observaron que a los 6 años de tratamiento con GH no hubo influencia en la recurrencia tumoral.

Por su parte, Sánchez ${ }^{17}$ realizó un estudio sobre tratamiento con hormona de crecimiento en la comunidad valenciana en niños con déficit de GH y en pequeños para la edad gestacional, donde se incluyó 5 pacientes con diagnóstico de craneofaringioma operado. En ninguno de ellos hubo recurrencia del tumor durante el tratamiento con $\mathrm{GH}$.

Un estudio multicéntrico realizado por Müller et al. ${ }^{18}$ no muestra diferencia en la recidiva y progresión tumoral entre pacientes operados de craneofaringioma, 54 pacientes que llevaron tratamiento con GH y 60 pacientes sin tratamiento, después de un seguimiento promedio de 3 ańos.

Olsson et al. $^{7}$ estudiaron a 126 pacientes con craneofaringioma operado, con y sin tratamiento con $\mathrm{GH}$, de los cuales obtuvieron una progresión o recidiva en $16 \%$ de los pacientes que recibieron $\mathrm{GH}$, en comparación con el $43 \%$ de los pacientes que no recibieron tratamiento con esta hormona. Concluyeron que el tratamiento con dosis fisiológicas de GH no influye en la progresión tumoral en pacientes con craneofaringioma.

Rodríguez et al. ${ }^{27}$ reportaron recientemente a 7 pacientes con craneofaringioma operado, con seguimiento por 15 ańos; 3 de ellos recibieron tratamiento con $\mathrm{GH}$, tras el cual observaron recidiva tumoral en 2 de ellos.
En Cuba, después del análisis de una casuística de 813 pacientes tratados con GH en el período 2005-2017, solo se encontró la paciente cuyo caso clínico se presentó en este trabajo, con diagnóstico de craneofaringioma y estudios imagenológicos (TAC) hipofisarios sin recidiva tumoral, que fue incorporada al tratamiento con $\mathrm{GH}$, por lo que es importante su presentación.

Con relación al caso clínico, la paciente recibió el tratamiento con hormona GH y realizó los controles médicos respectivos donde se evaluaron parámetros imagenológicos que descartaron recidiva tumoral, presentando una respuesta favorable al tratamiento con GH. Tomando en cuenta la experiencia en otros lugares, se puede concluir que, con una adecuada selección del paciente, con una demostración clínica e imagenológica de eliminación del proceso neoplásico $\mathrm{y}$ con un seguimiento por un equipo multidisciplinario, se puede orientar el tratamiento con GH en estos pacientes y no alejarlos de los beneficios de esta terapia.

\section{Bibliografía}

1. Kronenberg H, Melmed S, Polonsky K, Larse P. Williams textbook of endocrinology. $13^{\mathrm{a}}$ ed. Philadelphia: Elsevier; 2016.1936 p.

2. Pombo M, Audi L, Bueno M, Calzada F, Bergadá C, Diéguez C, et al. Tratado de endocrinología pediátrica. $4^{a}$ ed. Madrid: McGrawHill; 2009. 1028 p.

3. Farreras V, Rozman C. Medicina interna. Metabolismo y nutrición Endocrinología. 17 ed. Barcelona, España: Elsevier; 2014.312 p.

4. Kliegman R, Bonita F, Geme J, Schor N. Nelson textbook of pediatrics. $20^{a}$ ed. Philadelphia: Elsevier; 2016.3904 p.

5. Karavitaki N, Cudlip S, Adams CB, Wass JA. Craniopharyngiomas. Endocrine Reviews. 2006; 27(4): 371-397. 
6. Cucit S. Craneofaringioma. FASEN. 2008; 3(4): 12-22.

7. Olsson DS, Buchfelder M, Wiendieck K, Kremenevskaja N, Bengtsson BA, Jakobsson $\mathrm{KE}$, et al. Tumour recurrence and enlargement in patients with craniopharyngioma with and without $\mathrm{GH}$ replacement therapy during more than 10 years of follow-up. Eur J Endocrinol/ Eur Feder Endocr Soc. 2012 Jun; 166(6): 1061-1068.

8. Bergadá I. Utilización de la hormona de crecimiento en niños y adolescentes. Medicina (Buenos Aires). 2013; 73: 272-276.

9. Reiter EO, Price DA, Wilton P, Albertsson $\mathrm{K}$, Ranke MB. Effect of growth hormone (GH) treatment on the near-final height of 1258 patients with idiopathic GH deficiency: Analysis of a large international database. J Clin Endocrinol Metab. 2006; 91: 2047-54.

10. Wilton P. Adverse events reported in KIGS. In: Ranke MB, Price DA, Reiter EO, eds. Growth hormone therapy in Pediatrics, 20 years of KIGS. Basel: Karger. 2007. pp 432-441.

11. Calzada R. Uso de la hormona de crecimiento humana recombinante (HChr). Rev Med Inst Mex Seguro Soc. 2017; 55(2): 196-213.

12. Darendeliler F. Safety of Growth Hormone Treatment. J Clin Res Pediatr Endocrinol. 2009; 1: 36-43.

13. Samani AA, Yakar S, Leroith D, Brodt O. The role of the IGF system in cancer growth and metástasis overview and recent insights. Endocrine Reviews. 2007; 28: 20-47.

14. Pollak MN, Schernhammer ES, Hankinson SE. Insulin-like growth factors and neoplasia. Nature Reviews. Cancer. 2004; 4: 505-518.

15. Hernández Y, Jorge R. Deficiencia de la hormona de crecimiento en adultos. Diagnóstico y tratamiento. Rev Cubana Endocrinol. 1999; 10(3): 212-216.

16. Ciaccio M, Gil S, Guercio G, Vaiani E, Alderete D, Palladino M, Warman, et al. Evaluación de la talla final y sobrevida libre de eventos en pacientes sobrevivientes de meduloblastoma con deficiencia de hormona de crecimiento, tratados o no con hormona de crecimiento biosintética recombinante humana en la niñez. Comparación con pacientes con craneofaringioma tratados con rhGH. Medicina infantil. 2010 jun; 17(2): 121-128.

17. Sánchez J. Estudio sobre tratamiento con hormona de crecimiento en la comunidad valenciana en nińos con déficit de $\mathrm{GH}$ y en pequeńos para la edad gestacional (tesis doctoral). Valencia: Universidad de Valencia; 2014.

18. Muller HL, Gebhardt U, Schroder S, et al. Analyses of treatment variables for patients with chilhood craniopharygioma results of the multicenter prospective trial KRANIOPHARYNGEOM 2000 after three years of follow up. Horm Res Paediatrics. 2010; 73(3): 175-180.

19. Schoenle E, Zapf J, Prader A, Torresani T, Werder E, Zachmann M. Replacement of growth hormone $(\mathrm{GH})$ in normally growing $\mathrm{GH}$ deficient patients operated for craniopharyngioma. J Clin Endocrinol Metabolism. 1995; 80(2): 374-378.

20. Crowley R, Thompson C. Management of Craniopharyngioma-Perspectives beyond Surgery and Endocrinology. Eur Endocrinol. 2015; 11(2): 96-97.

21. Wait D. Lessons from the national cooperative growth study. J Eur Endocrinol. 2004; 151: 55-59.

22. Maneatis T, Baptista J, Connelly K, et al. Growth hormone safety update from the 
National Cooperative Growth Study. J Pediatr Endocrinol Metab. 2000; 13: 1035-1044.

23. Darendeliler F, Karagiannis G, Wilton P, Ranke MB, Albertsson-Wikland K, Anthony Price $\mathrm{D}$, et al. Recurrence of brain tumors in patients treated with growth hormone: analysis of KIGS (Pfizer International Growth Database). Acta pediátrica. 2006 oct; 95(10): 1284-1290.

24. Gonzáles I, Coral A. Seguridad del tratamiento con hormona de crecimiento: rhGH y cáncer. Rev Esp Endocrinol Pediatr. 2013; 4(1): 43-50.

25. Karavitaki N, Warner JT, Marland A, et al. $\mathrm{GH}$ replacement does not increase the risk of recurrence in patients with craniopharyngioma. Clin Endocrinol (oxf). 2006; 64(5): 556-560.

26. Karavitaki N, Brufani C, Warner JT, Adams CB, Richards P, Ansorge O, et al. Craniopharyngiomas in children and adults: Systematic analysis of 121 cases with long term follow up. Clinical Endocrinology. 2005; 62; 397-409.

27. Rodríguez F, Salamanca L, Guerrero J, Garcia P, Carceller B, Gonzalez I. Craneofaringioma en la edad pediátrica: experiencia en un hospital terciario. Rev Esp Endocrinol Pediatr. 2013; 4(1): 232-237. 